\title{
Work Measurement Study to Increasing Productivity of Production Line
}

\author{
Daniela Onofrejová1* , Jaroslava Janeková1, Juraj Šebo ${ }^{1}$ \\ ${ }^{1}$ Technical University of Kosice, Faculty of Mechanical Engineering, Institute of Management, Industrial and Digital Engineering, Park Komenskeho 9 , \\ 04200 Kosice, Slovakia
}

\begin{abstract}
The main goal of the paper is to propose solution toward capacity improvement of assembly process on a selected production line. The major content is focused on Basic Most method and its application in improvement process. There is a theoretical expectation that after applying Basic Most method to the assembly process, the capacity of production line would increase about twenty percentages. Motion and Time studies are often applied for improvements in efficiency of assembly processes. First, Process Screen method is used for gaining the information about process behaviour and its individual, partial and total duration. The final solution consists of new layout design of working station at the selected production line.
\end{abstract}

Keywords: basic MOST method; motion and time studies; capacity improvement; process behaviour; assembly process.

\section{Introduction}

The basic precondition for work improvement of an operator in an industrial plant is work analysis and measurement. In his book, Kubalak defines the analysis of work as: "analysis of individual work operations in terms of their scope, difficulty, duration and working conditions" [1]. Work measurement is specific part of work improvement process, usually interconnected with work analysis.

Work measurement is defined as "the application of techniques designed to determine the time that needs qualified personnel for execution of the specified work in certain technical and organizational conditions at the given level of performance" [2]. It provides management with a means to measure the time of an operation where effective and inefficient working time is displayed [3], [9].

Standard work measurement techniques are:

\section{$\checkmark$ Estimates,}

$\checkmark$ Direct methods,

$\checkmark$ Indirect methods.

Direct methods include such methods, which determine precise consumption of time and give a precise picture of the time distribution. In the literature, this group is also called time studies. On the basis of the length and continuity of time measurement the time studies could be divided into [4]:

\section{The continuous time studies}

2. The snapshot time studies.

Indirect methods determine precise consumption of time on the base of the operator's movements during his work. In the literature we can find them also as movement studies. There are two names used for these methods: Predetermined 
Motion Time System (PMTS) or Predetermined Time Standards (PTS). These methods are based on predetermined times of standard motions and the fact that work operations are composed of elementary movements [5]. The most suitable application is when the cycle time is low or middle and production volume is high or middle [6], [8]. The most well-known indirect methods are:

\section{MTM (Methods Time Measurement),}

2. MOST (Maynard Operation Sequence Technique),

3. MODAPTS (Modular Arrangement of Predetermined Time Standards).

MOST (Maynard Operation Sequence Technique) technique is one of the most productive methods of work measurement. This technique of work measurement is based on the movement of the objects and generalized experience. For calculation of operation time it uses 4 standard sequences of movement (general move, controlled move, tool use and hand crane use) which are composed of basic actions (reach, grasp, move, position the object, etc.). When applying the method, the numerical parameters are assigned to basic actions according to specific characteristics of the actions predefined in MOST tables. The final time is then calculated from the sum of assigned parameters. Since the final time is measured in a special unit called Time Measurement Unit (TMU), the conversion to regular time unit is needed (1 TMU $=0.036$ second). Visnansky [7] mentions following advantages of MOST technique: not needed stopwatches, suitable for each environment, quick application, accuracy and low costs.

Basic MOST is a medium degree MOST method. Its use is suggested in operations with 150 to 1500 repetitions per week and time length between 2-10 minutes [6], [8].

\section{Experimental Material and Methods}

\subsection{Analysis of an evaluated object - a final assembly line}

The major goal of the paper is to propose solution toward capacity improvement of assembly process on a selected production line. Motion and Time studies are often applied for improvements in efficiency of assembly processes [9], [10]. The major content is focused on Basic MOST method and its application in improvement process.

The studied production is performed in a production hall consisting of several production lines deployed to ensure the most efficient material flow. The manufacturing process consists of several inputs - components that are transformed into outputs - semi-finished products and final product so, that the final product is ready for shipping. The final products are primarily taken from the production hall to the warehouse and then sent to the customers. The outlined layout of the production hall (Figure 1) describes the flow of material for production lines within the company, as can be seen the material flow has a serial sequence in section 1. Final production line, that is a matter of study, is divided into three sections. Thus, outputs of each section would be examined and evaluated.

At the first part of the line, the main assembly operations are performed by ten operators and two screwdrivers. In this section, the entire product is assembled except the top cover. Screwdrivers are used to screw PCB boards to the player's bottom chassis. Due to the precision of screwing, torque

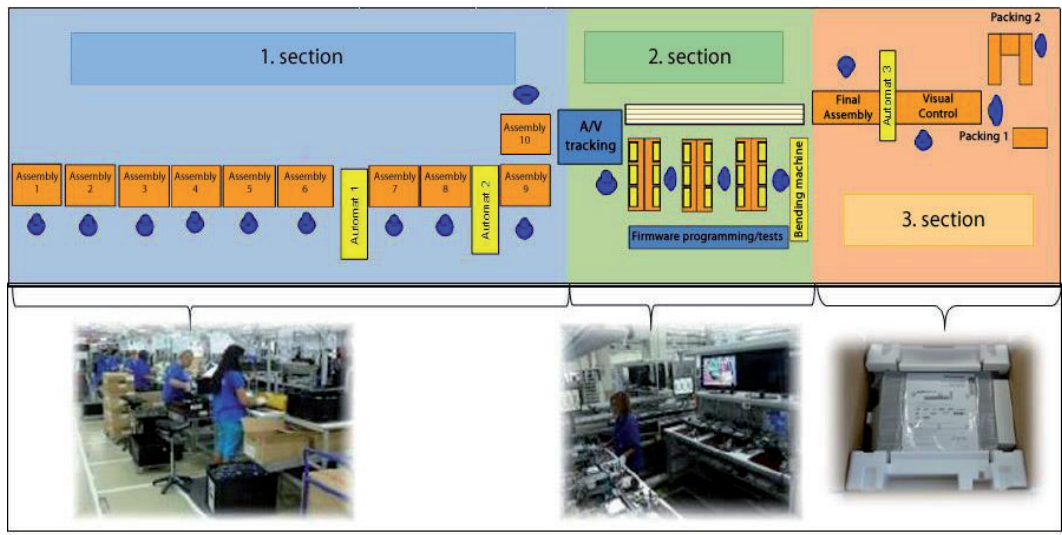

Fig. 1: Line arrangement of production line-location of the workplace is divided into three sections of the final line. 
compliance and screw driving speed, the machines are more advantageous than operators. In this way, errors such unscrewed or twisted screws can be avoided.

The second section of the line consists of three workplaces that are operated by four operators. At the first workplace an operation Audio/video scan is performed, and the product's operating program is burned using installation CDs or USB keys. The second workplace is the Inspection cell, which task is to check the functionality of the entire product, including image and sound testing. The last workplace is Bending operation, on which the player's top cover is bended by automatic bending machine, and is operated by one operator.

The third section consists of four workstations. The first one is the final assembly there a top moulded cover is placed on the product. Subsequently, the visual inspection of a final product is performed by an operator. All protective films are removed in this workplace and the product is ready for packaging into a cardboard box along with accessories such as user manuals, remote control, power cable and batteries. As the last operation, the palletizing of the product is performed by the operator, after which the product is ready for storage or dispatch. Altogether, 18 specialized operators work at the Final Line by 22 operating stations.

2.2. Basic MOST - method for analysis and measurement of work

Techniques for analysing and measuring work are a great tool to eliminate inefficiencies in performing work.

Analysis and Measurement of Work refers to a systematic review of workflows to improve resource efficiency (productivity) and define time standards for each activity.

Basic MOST method is a system for analysing, measuring and improving working processes; therefore, it focuses on the movement of objects. Movement can be done in two ways:

\section{$\checkmark$ objects are grasped and moved freely in a space,}

$\checkmark$ objects are moved in space so that they are in constant contact with any other surface.

For each movement, a string of other events occur, therefore Basic MOST uses a variety of activity sequence models (Table 1). In principle, only three Basic MOST activity sequences are needed to describe manual work, plus fourth to measure motion objects with manual crane. These are:

$\checkmark$ General displacement (relocation) sequence - free movement of an object in a space;

$\checkmark$ Sequence of Controlled Relocation - linked movement of the object in the space, remains in contact with another surface while moving, alternatively it is a part of another moving object;

$\checkmark$ Tool Usage Sequence - usage of common hand tools.

Table 1: Sub-activities arranged in a sequence model consisting of a series of parameters organized in a logical sequence.

\begin{tabular}{|c|c|c|c|}
\hline \multicolumn{4}{|c|}{ Sequence models for Basic MOST Work Measurement Technique } \\
\hline Activity & Sequence model & Parameter & Sub-activity \\
\hline \multirow{4}{*}{$\begin{array}{c}\text { General } \\
\text { Relocation }\end{array}$} & \multirow[t]{4}{*}{ ABGABPA } & A & Action distance \\
\hline & & B & Body motion \\
\hline & & G & Gain control \\
\hline & & $\mathrm{P}$ & Placement \\
\hline \multirow{2}{*}{$\begin{array}{l}\text { Controlled } \\
\text { Relocation }\end{array}$} & \multirow[t]{2}{*}{ ABGMXIA } & M & Move controlled \\
\hline & & $\begin{array}{l}x \\
I\end{array}$ & $\begin{array}{l}\text { Process time } \\
\text { Alignment }\end{array}$ \\
\hline \multirow{7}{*}{ Using a Tool } & \multirow[t]{7}{*}{ ABGABPFABPA } & $\mathrm{F}$ & Fasten \\
\hline & & $\mathrm{L}$ & Loosen \\
\hline & & C & Cut \\
\hline & & $S$ & Surface treat \\
\hline & & M & Measure \\
\hline & & R & Record \\
\hline & & $\mathrm{T}$ & Think \\
\hline
\end{tabular}

\section{Results and Discussion}

Measurements are made to optimize production costs before commencing serial production of the model in the development phase. At this stage, the individual operations and movements of the operator performed during product assembly are examined. Production time is analysed, bottlenecks are examined, and line performance standards are determined accordingly. Part of the measurement process is to provide a snapshot of the operation recorded in the checklist. The team leader measures each operation with a stopwatch or a video recorder. The analysed operation unit consists of 22 operation stations serviced by eighteen operators and three automatic machines arranged in a serial line.

Application of the Basic MOST method involves recording the number of movements in particular index into a recording sheet. The record contains the number of the operation, a detailed description of the performed movement at work, by which it is possible to determine the sequence of movement. Sequence classification is processed in the Table 1. Subsequently, predetermined index values are assigned to the sequences according to the motion. Conversion value for converting TMU into time measures is $1 \mathrm{TMU}=0.036 \mathrm{~s}$. 
Table 2: Analysis output of Basic MOST method for one working position.

\begin{tabular}{|c|c|c|c|c|c|c|c|c|c|c|c|c|c|c|c|c|c|c|c|}
\hline MOST & \multicolumn{19}{|c|}{ Bending machine } \\
\hline \multirow{2}{*}{\begin{tabular}{|l|} 
No. \\
\end{tabular}} & Description & \multicolumn{15}{|c|}{ Sequence } & \multirow{2}{*}{ 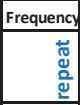 } & \multirow{2}{*}{\begin{tabular}{|r|} 
TMU \\
$\stackrel{2}{ }$ \\
\end{tabular}} & \multirow{2}{*}{\begin{tabular}{|r} 
time \\
\multirow{\Xi}{}{}
\end{tabular}} \\
\hline & Serial Line & A & B & G & A & B & $\mathbf{P}$ & M & $x$ & I & $\mathbf{F}$ & C & $\mathbf{S}$ & $\mathbf{T}$ & $\mathbf{R}$ & A & & & \\
\hline 1 & \multirow{6}{*}{$\begin{array}{l}\text { Take top cover from box } \\
\text { Clean and check } \\
\text { Turn top cover } 2 x \\
\text { Insert top cover in Bending machine } \\
\text { Take carton and place on a box } \\
\text { Press ON button }\end{array}$} & 3 & & 3 & & & & & & & & & & & & & 1.0 & 60 & 1.7 \\
\hline 2 & & & & & & & & & & & & & 6 & 10 & & & 1.0 & 160 & 4.6 \\
\hline 3 & & & & & 1 & & & 3 & & & & & & & & & 2.0 & 80 & 2.3 \\
\hline 4 & & & & & 1 & & 6 & & & & & & & & & & 1.0 & 70 & 2 \\
\hline 5 & & 1 & & 1 & 3 & & 1 & & & & & & & & & & 1.0 & 60 & 1.7 \\
\hline 6 & & 1 & & 1 & & & & 1 & & & & & & & & 3 & 1.0 & 60 & 1.7 \\
\hline \multirow{2}{*}{\multicolumn{18}{|c|}{ Total time consumption: }} & 490 & 14.1 \\
\hline & & & & & & & & & & & & & & & & & & TMU & $\mathrm{sec}$ \\
\hline
\end{tabular}

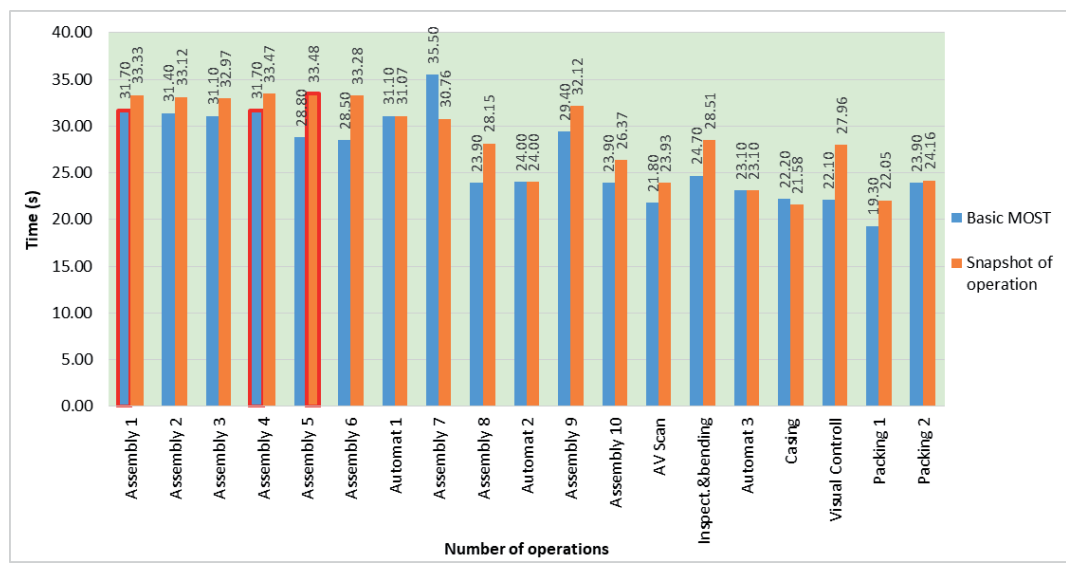

Fig. 2: Operation times measured using Snapshot method and Basic MOST methods for serial line.

Sequence A describes the distance; sequence $G$ describes gaining control over the object. The index value of distance $A$ is defined by index 3 - it means the distance of the object within 1-2 steps. Subsequently, the sequence index has to be assigned for $A 3$ and $G 3$. The simple calculation of the Basic MOST method for operation no.1 (Take top cover from box) is the sum of the index values $3+3$ and their multiplication by 10 , thus time in TMU is obtained. By multiplying the value of 0.036 seconds, the TMU is converted to seconds (Table 2). Sequence: $\mathrm{A} 3 \mathrm{G} 3=3+3=6 \times 10=60 \mathrm{TMU} \times 0.036 \mathrm{~s}$ $\mathrm{x}$ index $0.8=1.7 \mathrm{~s}$.

Measured time values by Snaphot method and Basic MOST method are compared, and divergences are identified from evaluated operations. It is obvious from Figure 2, that the time value for Assembly 5 is the highest, while in calculations using Basic MOST method it is the operation position Assembly 1 and Assembly 4. Here, proves by adjustment made by applying the Basic MOST method against the Snapshot time study is evident, and lower (better) values were achieved by Basic MOST method.

From the results, it can be determined that the critical point, even after the application of the Basic MOST method, is the first part of the line, where the lowest production amount is achieved (Figure 3). By applying the Basic MOST method, the increased production amount for the working shift was six percent (from 806 pieces to 852 pieces). Even the increase in production in the number of products was more about 46 pieces per shift, compared to the other parts of the line, the losses are still rather high. Therefore, there are problems in individual downtime between operations, since the first part of the line consists of 10 operators, it is clear that losses in downtime with the highest number of operators are unacceptable. The proposal for increasing effectiveness is modification of the workplace - the serial final production line. New design observes a change in the most critical point, and serial alignment was changed for parallel alignment of the workplace (Figure 4). This change assumes a shortening of downtime and thus achievement higher production quantities. 

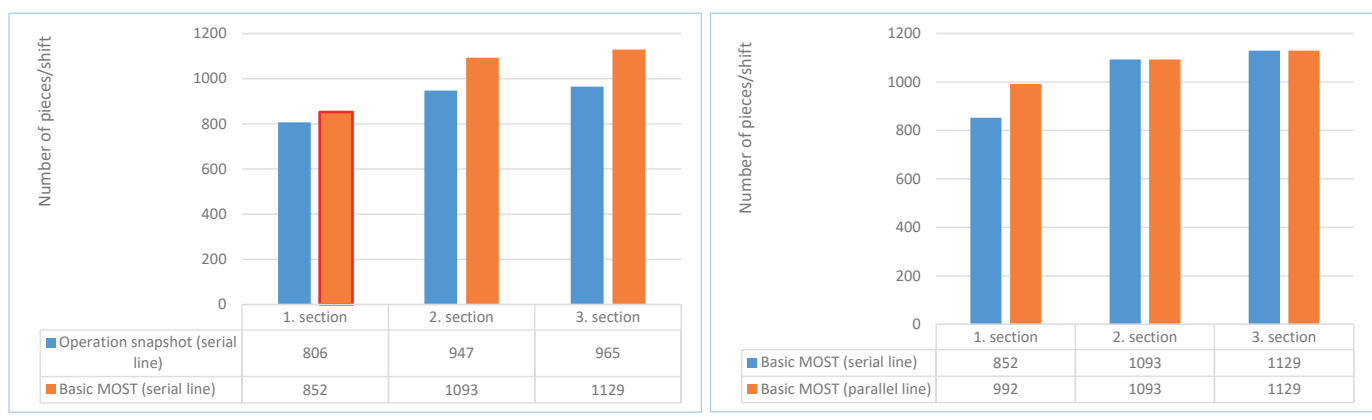

Fig. 3: Product quantity evolution after applying method Basic MOST (left) and change in line layout from serial to parallel (right).

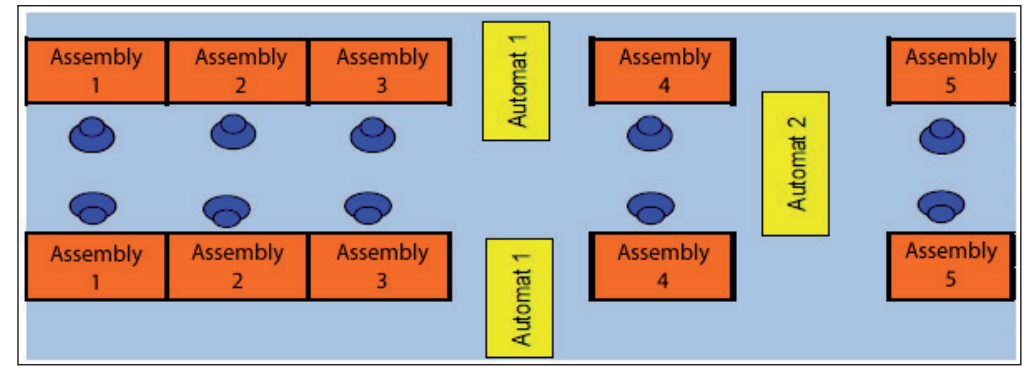

Fig. 4: Change in workplace layout - Parallel Line Design.

The number of units produced within the working shift signifies 140 pieces more after implementing the parallel design of the workplace. By applying the Basic MOST method for parallel line layout, the six percent increase in production volume (from 852 pieces to 992 pieces) was achieved compared to the Basic MOST method in a parallel workplace layout. After the new design of the workplace, the goal of the production quantity was achieved beyond the expectations. By applying the method Basic MOST, planned achievement in efficiency was $20 \%$ increase, and achieved results after implementation was a total of $23 \%$ efficiency.

\section{Conclusions}

The main goal was to increase the production volume per working shift for the final production line. The first step was to compare the snapshot measurement of the operation that is the real time measured with the Basic MOST method, which compares the predetermined time according to the movements at work. By comparing both methods, it was found that by using the Basic MOST method it is possible to achieve lower times of individual operations and thus higher production quantity. Based on the results of the Basic MOST method, operators should be able to perform operations at a given job at a lower time. Using a snapshot of the operation, one can determine the average time for a given operation, but cannot evaluate whether the resulting time is efficient enough. By applying the Basic MOST method, the increased production amount for the working shift was six percent (from 806 pieces to 852 pieces). Planned target was determined about 20 percent increase in production volume that is why the six percent threshold was reconsidered as insufficient. For this reason, it was necessary to proceed to the next step, changing the line layout from serial line to a parallel line alignment. The fact that the number of operators was not increased was taken into account in the design of the parallel line. Ten operators performed the final assembly on the serial line. The result of this serial deployment was a high number of downtime between operators, resulting in a lack of line efficiency. When designing the parallel line layout, the number of operations per operator was increased about two more steps in a procedure, so that the final assembly was performed by five operators and the downtime was reduced.

\section{Acknowledgments}

This article has been supported by the grant project KEGA 026TUKE-4/2017 "Implementation of innovative educational approaches and tools to enhance the development of the core competencies graduate study program Industrial Engineering" 
and Slovak Grant Agency VEGA 1/0708/16 "Development of a new research methods for simulation, assessment evaluation and quantification of advanced methods of production".

\section{References and Notes}

[1] Kubalak, M. (2013). Effective human resource management. Eurokodex, Zilina. (in Slovak)

[2] Zandin,B.K. (2003). MOST -Work Measurement Systems. CRC Press, Taylor \& Francis Group, Boca Raton, FL.

[3] Visnansky,M. et al. (2010) Analysis, measurement and standardization of work. IPA Slovakia., Zilina. (in Slovak)

[4] Stusek, J. (2007). Operations management in logistics chains. C.H. Beck pro praxi, Praha. (in Czech)

[5] Groover, M.P. (2006). Work Systems and the Methods, Measurement, and Management of Work. Prentice Hall, London.

[6] Kristak,J. et al. (2009). Analysis and measurement of work. IPA Slovakia, Zilina. (in Slovak)

[7] Visnansky, M. (2010). Basic MOST (lean workshops), IPA Slovakia, Zilina. (in Slovak)

[8] Kosturiak, J., Kristak, J., Kysel, M., Bednar, R. (2005). Analysis and measurement of work. IPA Slovakia, Zilina.

[9] Hitka, M. (2007). Working day snapshot. Available at internet: www.miloshitka.szm.sk/cop_2007.doc, 2016-04-10.

[10] Szombathyová, E., Krauszová, A. (2014) Ergonomic process and its application in practice. In: Management of Enterprises. Vol. 4, No. 2, pp. 132-136.

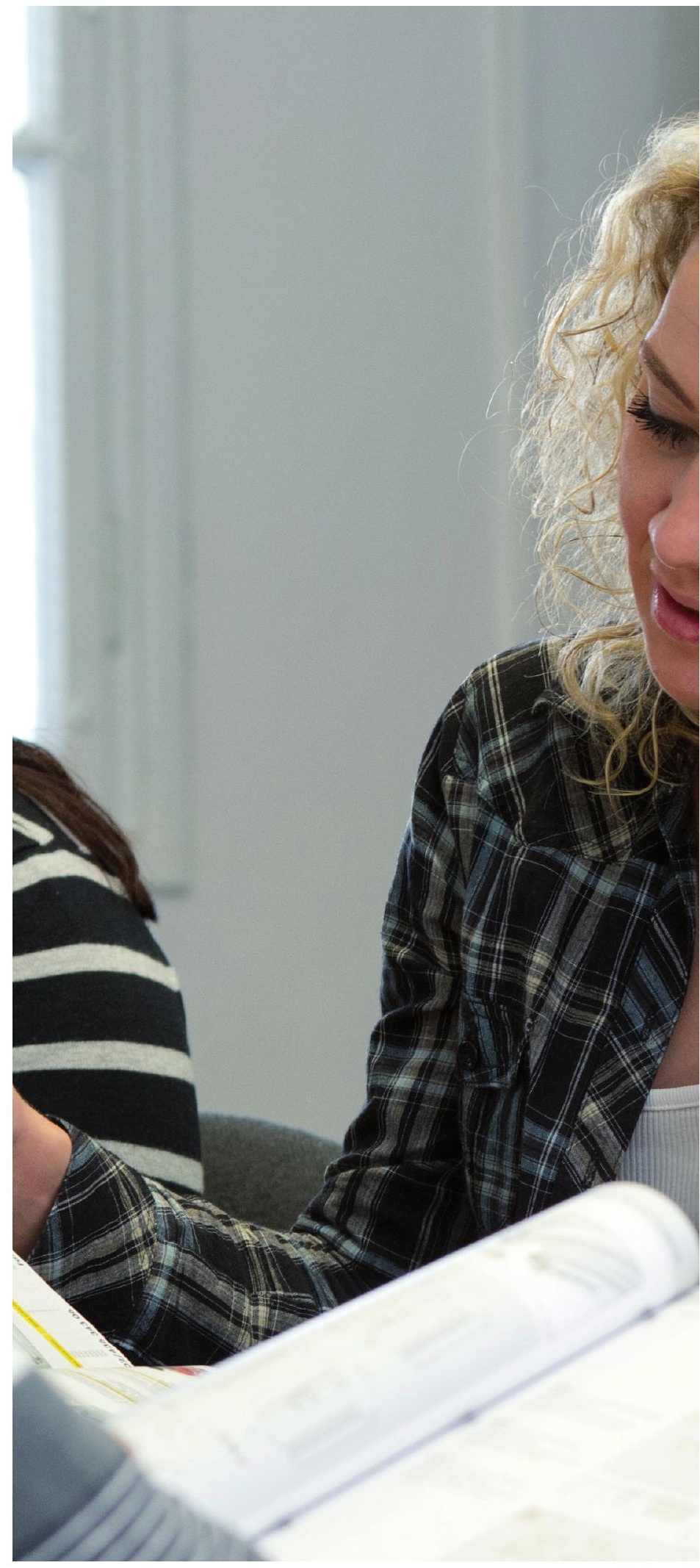

